\title{
Importance of asymptotic freedom for the pseudocritical temperature in magnetized quark matter
}

\author{
R. L. S. Farias,,$^{1,2, *}$ K. P. Gomes, ${ }^{1, \dagger}$ G. Krein,,${ }^{3, \ddagger}$ and M. B. Pinto ${ }^{4, \S}$ \\ ${ }^{1}$ Departamento de Ciências Naturais, Universidade Federal de São João Del Rei, 36301-000, São João Del Rei, MG, Brazil \\ ${ }^{2}$ Departamento de Física, Universidade Federal de Santa Maria, 97105-900, Santa Maria, RS, Brazil \\ ${ }^{3}$ Instituto de Física Teórica, Universidade Estadual Paulista, Rua Dr. Bento Teobaldo Ferraz, 271 - Bloco II, 01140-070 São Paulo, SP, Brazil \\ ${ }^{4}$ Departamento de Física, Universidade Federal de Santa Catarina, 88040-900 Florianópolis, Santa Catarina, Brazil
}

(Received 23 April 2014; revised manuscript received 17 June 2014; published 11 August 2014)

\begin{abstract}
Although asymptotic freedom is an essential feature of QCD, it is absent in effective chiral quark models like the Nambu-Jona-Lasinio and linear sigma models. In this work we advocate that asymptotic freedom plays a key role in the recently observed discrepancies between results of lattice QCD simulations and quark models regarding the behavior of the pseudocritical temperature $T_{p c}$ for chiral-symmetry restoration in the presence of a magnetic field $B$. We show that the lattice predictions that $T_{p c}$ decreases with $B$ can be reproduced within the Nambu-Jona-Lasinio model if the coupling constant $G$ of the model decreases with $B$ and the temperature. Without aiming at numerical precision, we support our claim by considering a simple ansatz for $G$ that mimics the asymptotic-freedom behavior of the QCD coupling constant $1 / \alpha_{s} \sim \ln \left(e B / \Lambda_{\mathrm{QCD}}^{2}\right)$ for large values of $B$.
\end{abstract}

DOI: 10.1103/PhysRevC.90.025203

PACS number(s): 21.65.Qr, 25.75.Nq, 11.30.Rd, 11.10.Wx

\section{INTRODUCTION}

The investigation of the effects produced by a magnetic field in the phase diagram of strongly interacting matter became a subject of great interest in recent years. The recent motivation stems mainly from the fact that strong magnetic fields may be produced in noncentral heavy-ion collisions [13]-for an updated discussion, see Ref. [4]. Strong magnetic fields are also present in magnetars [5,6] and might have played an important role in the physics of the early universe [7]. At vanishing baryon density and magnetic field, lattice QCD simulations $[8,9]$ predict that there is a crossover transition at a pseudocritical temperature $T_{p c}$. More recent lattice simulations [10-13] indicate that such a crossover persists in the presence of a magnetic field and, in addition, confirm predictions made a few years ago [14-16] of the phenomenon of magnetic catalysis (MC), in that the quark condensate is enhanced in the presence of the magnetic field at vanishing temperatures. These lattice results also lend support to calculations using effective models, e.g., those of Refs. [17-21]. There are, however, marked disagreements between lattice results and model calculations regarding the dependence of $T_{p c}$ on the strength $B$ of the magnetic field. Specifically, the lattice results of Refs. [12,13], performed with $2+1$ quark flavors and physical pion-mass values, predict an inverse catalysis, in that $T_{p c}$ decreases with $B$, while effective models predict an increase of $T_{p c}$ with $B$-an exception is a calculation [22] based on the MIT bag model which, on the other hand, misses the crossover nature of the transition. Earlier model evaluations have been mainly performed considering the two flavor Nambu-JonaLasinio model (NJL) [23] and the linear sigma model [17] within the mean-field approximation (MFA). Subsequently,

\footnotetext{
*ricardofarias@ufsj.edu.br

${ }^{\dagger}$ karinaponcianogomes@gmail.com

${ }^{\ddagger}$ gkrein@ift.unesp.br

${ }^{\S}$ marcus.benghi@ufsc.br
}

these evaluations have been considered at more sophisticated levels by coupling degrees of freedom related to the Polyakov loop (PNJL models) [18] and including strangeness [19]. Calculations have also performed beyond the MFA by using the functional renormalization group [24,25]. Nevertheless, despite those refinements in model calculations, no qualitative changes in the behavior of $T_{p c}$ with $B$ have been observed.

Possible explanations for the disagreement concerning the dependence of $T_{p c}$ on $B$ have been recently given in Refs. [2629]. A particularly insightful discussion is the one of Ref. [28]: the authors of this reference argue that the inverse catalysis is the result of the back reaction of the gluons due to the coupling of the magnetic field to the sea quarks. Such a back reaction is naturally implemented in the so-called entangled PNJL model [30,31], in that the four-quark coupling of the NJL model is made dependent on the Polyakov loop. Although such a model is also unable [32] to produce inverse catalysis, a very recent study [33] has shown that, if the lattice data [13] are fit by making the pure-gauge critical temperature $T_{0}$, a parameter of PNJL models, depend on $B$, the model gives inverse catalysis. A similar recent study [34] in the context of a quark-meson (QM) model has concluded that the only way to get inverse catalysis would be to let the Yukawa quark-meson coupling increase with $B$, but no qualitative agreement with lattice simulations for the phase diagram $T_{p c}(B)$ is obtained. Moreover, the situation does not improve when this model is extended with the Polyakov loop (PQM) and the model parameter $T_{0}$ is made $B$ dependent like in Ref. [33].

From this discussion, it is apparent that the failure of effective models in providing inverse catalysis can be traced to a more fundamental level by recalling that the couplings in those models do not run with the magnetic field, in contrast with QCD whose coupling, as Miransky and Shovkovy have shown [35], decreases for large $B$ in a manifestation of the asymptotic-freedom phenomenon. Therefore, in the present work we make use of an ansatz that makes $G$ a running coupling with $B$ and $T$, very much like the strong-coupling runs in QCD. As a result, we will show that, at $T=0$, the 
model realizes magnetic catalysis, and at $T \neq 0$, it realizes inverse catalysis, in qualitative agreement with the lattice simulations of Refs. [12,13]. For zero magnetic field, the idea of using an ansatz to mimic high-temperature lattice results for the effective QCD coupling within the NJL model is not new; it was implemented in Ref. [36] to study the decoupling of the pion at high temperatures. A similar ansatz was used very recently in Ref. [37] in a study of the quark spectral density in a strongly coupled quark-gluon plasma in the context of the QCD Dyson-Schwinger equations at finite temperature and density-for a review, see Ref. [38].

Our motivation is easily understood by recalling that, in the NJL model, the quark mass gap and the quark condensate are related by $M \sim-G\left\langle\bar{\psi}_{f} \psi_{f}\right\rangle$, where $\left\langle\bar{\psi}_{f} \psi_{f}\right\rangle$ grows with $B$, as a manifestation of MC. When $G$ does not run with $B$ and $T$, the quark mass gap and $T_{p c}$ also grow with $B$. One way to circumvent this problem has been recently suggested by Kojo and $\mathrm{Su}$ [27], who consider an effective interaction with infrared enhancement and ultraviolet suppression. Our proposal is that basically the same effect can be obtained by assuming that the NJL quark-quark coupling decreases with $B$ and $T$, as does the QCD coupling. As a result, the NJL model results turn out to be in qualitative agreement with the recent lattice results; the condensate grows in accordance with $\mathrm{MC}$ and the pseudocritical temperature for the chiral transition decreases with $B$. Although this agreement comes out in a particular model implementation, it lends support to to the suggestion that the inverse catalysis is the result of the back reaction of the sea quarks to the magnetic field [28].

On a wider perspective, the motivation for tuning highly simplified models of the NJL type to reproduce the decreasing of $T_{p c}$ with $B$ obtained from much more fundamental nonperturbative lattice QCD calculations comes within the same spirit as in previous studies using such models. The situation here is similar to the calculation of the hadron masses using lattice QCD simulations: while presently correct numbers for the masses are generated by the simulations, there is no information on the physical mechanisms that generate those numbers from gluon and (essentially massless) quark fields. In this respect, the NJL model, in particular, provides physical insight into the mechanism behind mass generation via dynamical chiral-symmetry breaking. In the present context, one is able to understand the decreasing of $T_{p c}$ with $B$ by using a magnetic-field-induced running of $G$, which is interpreted as being due to the indirect coupling of $B$ to the gluons whose physics is similar to that of asymptotic freedom.

The paper is organized as follows: In the next section we discuss the quark condensate considering the two-flavor NJL model for hot and magnetized quark matter in the MFA. The running of the coupling $G$ is introduced in the same section. Then, in Sec. III, we discuss our numerical results while our conclusions and perspectives are presented in Sec. IV.

\section{RUNNING COUPLING IN MAGNETIZED NAMBU-JONA-LASINIO MODEL}

The standard two flavor NJL model is defined by a fermionic Lagrangian density given by [39]

$$
\mathcal{L}_{\mathrm{NJL}}=\bar{\psi}(i \not \partial-m) \psi+G\left[(\bar{\psi} \psi)^{2}-\left(\bar{\psi} \gamma_{5} \vec{\tau} \psi\right)^{2}\right],
$$

where $\psi$ represents a flavor iso-doublet ( $u$ and $d$ flavors) and $N_{c}$-plet of quark fields, while $\vec{\tau}$ are isospin Pauli matrices-a sum over flavor and color degrees of freedom is implicit. The Lagrangian density in Eq. (2.1) is invariant under (global) $\mathrm{U}(2)_{\mathrm{f}} \times \mathrm{SU}\left(N_{c}\right)$ and, when $m=0$, the theory is also invariant under chiral $\mathrm{SU}(2)_{L} \times \mathrm{SU}(2)_{R}$. Within the NJL model a sharp cutoff $\Lambda$ is generally used as an ultraviolet regulator and, since the model is not renormalizable, one has to fix $\Lambda$ to physical quantities. The phenomenological values of quantities such as the pion mass $m_{\pi}$, the pion decay constant $f_{\pi}$, and the quark condensate $\left\langle\bar{\psi}_{f} \psi_{f}\right\rangle$ are used to fix $G, \Lambda$, and $m$. Here, we choose the set $\Lambda=650 \mathrm{MeV}$ and $G=5.022 \mathrm{GeV}^{-2}$ with $m=5.5 \mathrm{MeV}$ in order to reproduce $f_{\pi}=93 \mathrm{MeV}, m_{\pi}=$ $140 \mathrm{MeV}$, and $\left\langle\bar{\psi}_{f} \psi_{f}\right\rangle^{1 / 3}=-250 \mathrm{MeV}$ in the vacuum.

The expressions for the thermodynamic potential of the model in the MFA are well documented in the literature; see, e.g., Refs. [23,40]. For results beyond the MFA, see Ref. [41]. Thus, we refrain from repeating them here and start with the expression for the gap equation [21]:

$$
M_{f}=m_{f}-2 G \sum_{f}\left\langle\bar{\psi}_{f} \psi_{f}\right\rangle
$$

where $\left\langle\bar{\psi}_{f} \psi_{f}\right\rangle$ represents the quark condensate of flavor $f$

$$
\begin{aligned}
\left\langle\bar{\psi}_{f} \psi_{f}\right\rangle= & -\frac{N_{c} M_{f}}{2 \pi^{2}}\left\{\Lambda \sqrt{\Lambda^{2}+M_{f}^{2}}-\frac{M_{f}^{2}}{2} \ln \left[\frac{\left(\Lambda+\sqrt{\Lambda^{2}+M_{f}^{2}}\right)^{2}}{M_{f}^{2}}\right]\right\} \\
& -\frac{N_{c} M_{f}}{2 \pi^{2}}\left|q_{f}\right| B\left\{\ln \left[\Gamma\left(x_{f}\right)\right]-\frac{1}{2} \ln (2 \pi)+x_{f}-\frac{1}{2}\left(2 x_{f}-1\right) \ln \left(x_{f}\right)\right\} \\
& +\frac{N_{c} M_{f}}{2 \pi^{2}} \sum_{k=0}^{\infty} \alpha_{k}\left|q_{f}\right| B \int_{-\infty}^{\infty} \frac{d p_{z}}{E_{p, k}(B)}\left\{\frac{1}{e^{\left[E_{p, k}(B)\right] / T}+1}\right\},
\end{aligned}
$$

where $E_{p, k}(B)=\left(p_{z}^{2}+2 k\left|q_{f}\right| B+M_{f}^{2}\right)^{1 / 2}, x_{f}=M_{f}^{2} /\left(2\left|q_{f}\right|\right.$ $B$ ), and $\alpha_{k}=2-\delta_{0 k}$. In addition, $\left|q_{f}\right|$ is the absolute value of the quark electric charge; $\left|q_{u}\right|=2 e / 3,\left|q_{d}\right|=e / 3$, with $e=$ $1 / \sqrt{137}$ representing the electron charge-we use Gaussian 
natural units where $1 \mathrm{GeV}^{2}=1.44 \times 10^{19} \mathrm{G}$. Note also that here we have taken the chemical equilibrium condition by setting $\mu_{u}=\mu_{d}=\mu$. Details of the manipulations leading to this equation can be found in the appendix of Ref. [23]. Note that the condensates for the flavors $u$ and $d$ are different due to their different electric charges. Remark also that, in principle, one should have two coupled gap equations for the two distinct flavors: $M_{u}=m_{u}-2 G\left(\left\langle\bar{\psi}_{u} \psi_{u}\right\rangle+\left\langle\bar{\psi}_{d} \psi_{d}\right\rangle\right)$ and $M_{d}=m_{d}-$ $2 G\left(\left\langle\bar{\psi}_{u} \psi_{u}\right\rangle+\left\langle\bar{\psi}_{d} \psi_{d}\right\rangle\right)$. However, in the two-flavor case, the different condensates contribute to $M_{u}$ and $M_{d}$ in a symmetric way and, since $m_{u}=m_{d}=m$, one can write $M_{u}=M_{d}=M$.

Reference [13] presents results for $\left(\Sigma_{u}+\Sigma_{d}\right) / 2$ and $\Sigma_{u}-$ $\Sigma_{d}$, where $\Sigma_{f}=\Sigma_{f}(B, T)$ is defined as

$$
\Sigma_{f}(B, T)=\frac{2 m_{f}}{m_{\pi}^{2} f_{\pi}^{2}}\left[\left\langle\bar{\psi}_{f} \psi_{f}\right\rangle-\left\langle\bar{\psi}_{f} \psi_{f}\right\rangle_{0}\right]+1,
$$

with $\left\langle\bar{\psi}_{f} \psi_{f}\right\rangle_{0}$ being the quark condensate at $T=0$ and $B=0$.

Let us recall the important result by Miransky and Shovkovy [35] that, for sufficiently strong magnetic fields, $e B \gg \Lambda_{\mathrm{QCD}}^{2}$, the leading-order running of the QCD coupling constant $\alpha_{s}$ is given by

$$
\frac{1}{\alpha_{s}} \sim b \ln \frac{e B}{\Lambda_{\mathrm{QCD}}^{2}},
$$

where $b=\left(11 N_{c}-2 N_{f}\right) /(12 \pi)$, and the energy scale $\sqrt{e B}$ is fixed up to a factor of order 1 . Motivated by this result, we propose for the NJL coupling, at $T=0$, the interpolating formula

$$
G(B)=\frac{G_{0}}{1+\alpha \ln \left(1+\beta \frac{e B}{\Lambda_{\mathrm{QCD}}^{2}}\right)},
$$

with $G_{0}=5.022 \mathrm{GeV}^{-2}$, which is the value of the coupling at $B=0$. The free parameters $\alpha$ and $\beta$ are fixed to obtain a reasonable description of the lattice average $\left(\Sigma_{u}+\Sigma_{d}\right) / 2$ for $T=0$. In principle, for the values of $B$ presently considered in lattice simulations, there is no reason for $G(B)$ to have a logarithm-running like the large- $B$ running of $\alpha_{s}(B)$. The proposed parametrization for the running of $G$ with $B$ is motivated, primarily, by the assumption that similar physics that drives asymptotic freedom is also responsible for the decrease of the effective coupling with the magnetic field; namely, the back reaction of gluons via the coupling of the magnetic field on the sea quarks. In addition, although the precise $B$ dependence of the effective coupling for values of $e B$ not much larger that $\Lambda_{\mathrm{QCD}}^{2}$ is not known, the proposed parametrization involves a minimum number of fitting parameters, extrapolates smoothly to the large- $B$ running of the quark-gluon running in QCD, and can be tested when larger values of $B$ will be used in lattice simulations. As we will show in the following, only a decrease (not necessarily in a logarithmic way) of $G$ with $B$ can account for the recent lattice results at $T=0$.

At high temperatures, $\alpha_{s}$ also runs as the inverse of $\ln \left(T / \Lambda_{\mathrm{QCD}}\right)$. However, the values of $T$ used in the lattice simulations of Refs. [12,13], $T \leqslant \Lambda_{\mathrm{QCD}}$, are not high enough to justify the use of such a running for $G$. Since the explicit form in which $\alpha_{s}$ runs with $B$ and $T$ is presently not known,

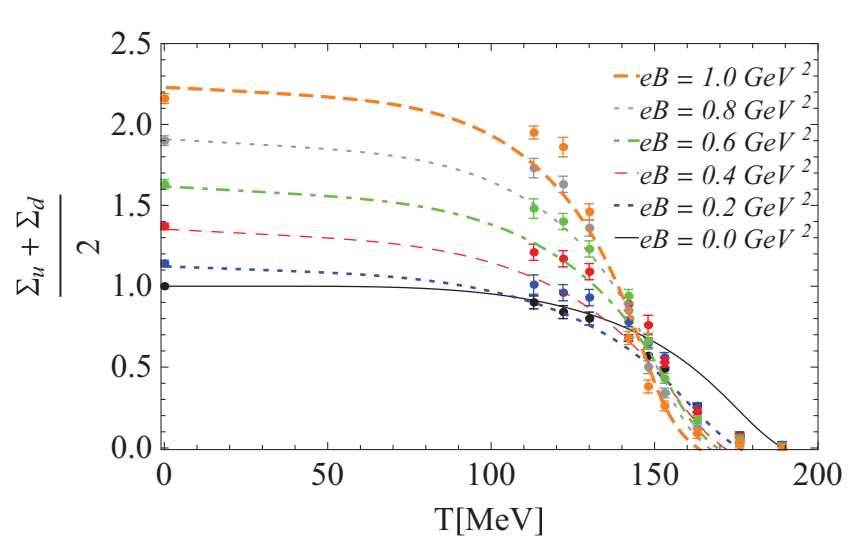

FIG. 1. (Color online) The condensate average as a function of the temperature. Data points are from the lattice simulations of Ref. [13].

we consider a $T$ dependence for $G$ as

$$
G(B, T)=G(B)\left(1-\gamma \frac{|e B|}{\Lambda_{\mathrm{QCD}}^{2}} \frac{T}{\Lambda_{\mathrm{QCD}}}\right),
$$

where $\gamma$ is fixed to obtain a reasonable description of the temperature dependence of the lattice average $\left(\Sigma_{u}+\Sigma_{d}\right) / 2$ at the highest temperatures. Notice that terms proportional to $T$, $T^{2},(B T)^{2}, \ldots$ could be considered; however, it turned out that they are not needed to obtain a reasonable fit to the lattice data for $\left(\Sigma_{u}+\Sigma_{d}\right) / 2$. It should be clear that, for the values of $B$ used in the lattice simulations, any function $G(B)$ that gives an effective coupling that decreases with $B$ can fit the lattice data for $\left(\Sigma_{u}+\Sigma_{d}\right) / 2$ for the magnetic-field values used in the simulations if a sufficient number of free parameters are used.

\section{NUMERICAL RESULTS}

Before presenting our results, we note that Refs. [12,13] used $m_{u}=m_{d}=5.5 \mathrm{MeV}, m_{\pi}=135 \mathrm{MeV}$, and $f_{\pi}=$ $86 \mathrm{MeV}$ in the multiplicative factor $m_{f} /\left(m_{\pi}^{2} f_{\pi}^{2}\right)$ in Eq. (2.4) to make $\Sigma_{f}(B, T)$ dimensionless. The fact that these values differ from the ones we use is of no significance for comparison purposes since they only set a general scale. We also consider $\Lambda_{\mathrm{QCD}}=200 \mathrm{MeV}$.

Figure 1 displays the condensate average $\left(\Sigma_{u}+\Sigma_{d}\right) / 2$. We obtain a good fit of the data at $T=0$ with $\alpha=2$ and $\beta=0.000327$ in Eq. (2.6). Figure 1 reveals that the magnetic catalysis is naturally reproduced at $T=0$. In addition, the $T$ dependence is also reasonably well reproduced-here we used $\gamma=0.0175$ in Eq. (2.7). Having fixed our parameters, we proceed in the analyses of other quantities.

The difference $\Sigma_{u}-\Sigma_{d}$ is displayed in Fig. 2. Although there is a small deviation from the lattice results for the highest values of $B$, the overall agreement is quite impressive, given the simplicity of the model.

In this work we consider the physical point with nonzero current quark masses so that, at high temperatures, the model displays a crossover where chiral symmetry is partially restored. In this case, one can only establish a pseudocritical temperature which depends on the observable used to define 


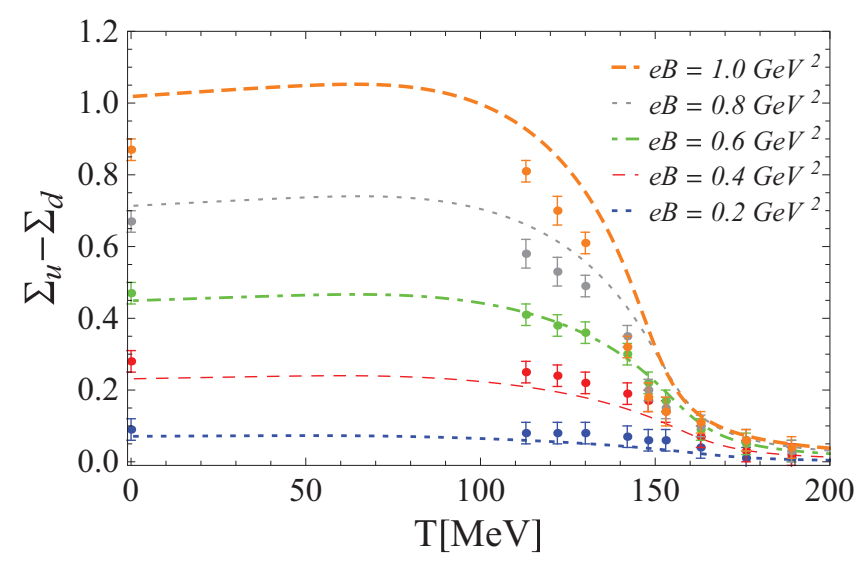

FIG. 2. (Color online) The condensate difference as a function of temperature. Data points are from the lattice simulations of Ref. [13].

it. Here, we use the location of the peaks for the vacuum normalized quark condensates, where the thermal susceptibilities are given by

$$
\chi_{T}=-m_{\pi} \frac{\partial \sigma}{\partial T},
$$

with $\sigma$ being defined by

$$
\sigma=\frac{\left\langle\bar{\psi}_{u} \psi_{u}\right\rangle(B, T)+\left\langle\bar{\psi}_{d} \psi_{d}\right\rangle(B, T)}{\left\langle\bar{\psi}_{u} \psi_{u}\right\rangle(B, 0)+\left\langle\bar{\psi}_{d} \psi_{d}\right\rangle(B, 0)} .
$$

In Fig. 3 we plot the thermal susceptibility defined by Eq. (3.1) as a function of the temperature for different values of the magnetic field. The figure clearly indicates the decrease of $T_{p c}$ for increasing values of the magnetic field. Again, the effects of asymptotic freedom seem to be a rather important feature to conciliate results obtained with the NJL model and lattice simulations.

Finally, in Fig. 4 we present the results for the pseudocritical $T_{p c}$ temperature as a function of the magnetic field. It is clearly seen in this figure that the pseudocritical temperature

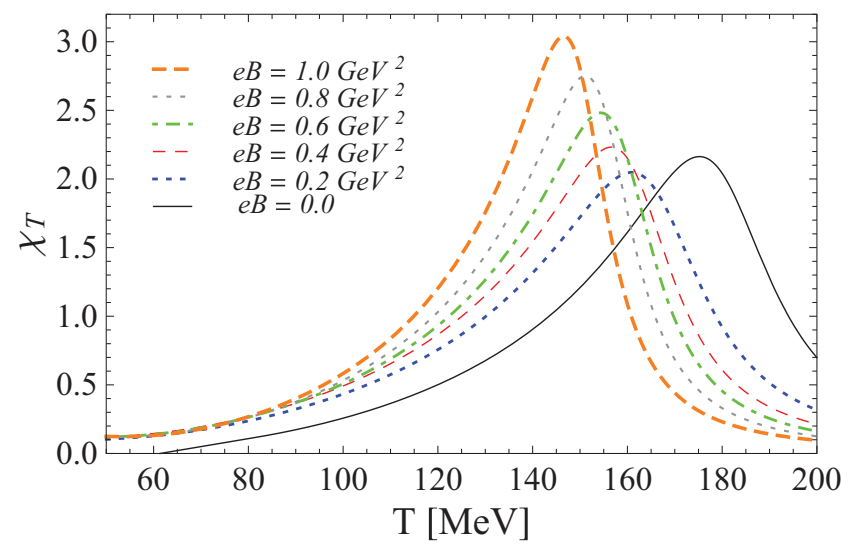

FIG. 3. (Color online) The normalized thermal susceptibility as a function of the temperature for different values of the magnetic field $B$.

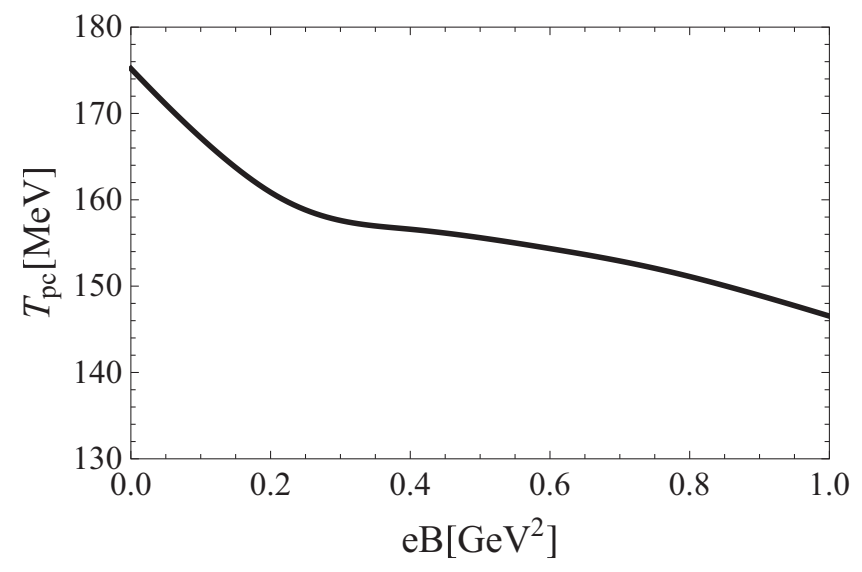

FIG. 4. The pseudocritical temperature for the chiral transition of magnetized quark matter as a function of magnetic field.

decreases as $B$ increases, in (qualitative) agreement with the lattice results of Refs. [12,13].

The phase diagram shown in Fig. 4 reproduces qualitatively the lattice results. The figure shows a $T_{p c}$ dependence with $B$ that starts linearly at small $B$, with almost no curvature, while the lattice results have an almost-vanishing linear term and a noticeable curvature. One may view this as a consequence of the rather simple form used for the running of $G(B, T)$. However, it is important to note that our ansatz avoids the undesired "turnover" effect seen in NJL models with a $B$ independent coupling, in which at intermediate values of $B$, after an initial decrease, $T_{p c}$ starts to increase with $B$ - see, e.g., Refs. [33,34]. A better quantitative agreement with the lattice phase diagram can certainly be obtained by using a $G(B, T)$ with more fitting parameters.

\section{CONCLUSIONS AND PERSPECTIVES}

In this work we have considered the two-flavor NJL model for hot and magnetized quark matter within the MFA. With the aim of understanding discrepancies between effective model predictions and recent lattice results regarding the behavior of the chiral-transition pseudocritical temperature as a function of $B$, we examined the effect of introducing a running coupling $G$ motivated by asymptotic freedom. Effective quark theories such as the NJL model can be motivated by QCD by integrating-out gluonic degrees of freedom. Although some features of confinement can be enforced by means of extending the model with the Polyakov loop, the running with energy scales of the effective coupling, such as, e.g., due to asymptotic freedom, is lost. In general, a decrease of $G$ with the temperature does not modify the qualitative features regarding the chiral phase transition [36]. However, as we have discussed, the same does not seem to be the case in the presence of a strong magnetic field when the formation of a quark condensate $\left\langle\bar{\psi}_{f} \psi_{f}\right\rangle$ is enhanced by the magnetic catalysis effect. Generically, the quark mass gap at zero temperature sets the pseudocritical temperature at which chiral symmetry is (partially) restored. Since the effective quark mass $M$ in the NJL model, given by the gap equation $M \sim-G\left\langle\bar{\psi}_{f} \psi_{f}\right\rangle$, 
increases with $B$, one would intuitively expect that $T_{p c}$ should also increase; but this is not confirmed by lattice simulations. Here, we have shown that one way to conciliate the results is to suppose that $G$ decreases with $B$ and $T$ so as to moderate the increase of $M$ and, in turn, to the decrease of $T_{p c}$ with $B$ in qualitative agreement with the $T_{p c}(B)$ obtained by the lattice simulations of Refs. [12,13].

As future work, one can improve the ansatz so as to get a better quantitative agreement with the lattice results. Next, the model could be used in a plethora of situations in order to analyze the behavior of physical quantities such as decay constants, meson-quark couplings, among others which can be tested in lattice simulations. One could also extend the calculations to the finite-chemical-potential domain, which currently is inaccessible to lattice calculations, and investigate eventual additional running of $G$ with $\mu$. This is particularly interesting, because a recent study has shown [42] that the coexistence chemical potential decreases with $B$. Such a study is important in the context of the physics of magnetars.

Also, it would be interesting to further investigate why the results for $T_{p c}(B)$ obtained with $G(B, T)$ and $T_{0}(B)$ in the NJL and entangled PNJL models, respectively, are in qualitative agreement with the QCD lattice results while those obtained with the QM and PQM models are not. Another interesting new perspective is the investigation of the effect of a $G(B, T)$ in the generation of spin-one condensates [43]. Such spin-one condensates give rise to a dynamical anomalous magnetic moment for the fermions and lead to an increased critical temperature for chiral symmetry restoration for $B$ and $T$ independent NJL coupling.

In conclusion, our assumption of the decrease of the effective four-quark coupling with $B$ and $T$ mimicking asymptotic freedom in QCD [35] represents a concrete implementation of the back reaction of the sea quarks and confirms its potential importance on explaining the inverse catalysis as stressed in the recent literature $[27,28]$. In this respect, a first-principles nonperturbative framework to study the effects of a magnetic field in the continuum is provided by the Dyson-Schwinger equations at finite temperature and density [38]. The coupling of the magnetic field to the sea quarks is naturally taken into account via the coupled system of integral equations for the quark and gluon propagators. Very recently, this approach was used to study fermion mass generation in the presence of an external magnetic field in QED [44,45] and QCD [27,46,47]. The extension of such studies to finite temperatures would shadow light on the problem of inverse magnetic catalysis beyond the framework of effective chiral models.

\section{ACKNOWLEDGMENTS}

We thank Gergely Endrodi for discussions and also for providing the lattice data of the up- and down-quark condensates. We also thank Eduardo Fraga for bringing Ref. [34] to our attention. This work was partially supported by $\mathrm{CNPq}$, FAPEMIG (R.L.S.F), FAPESP (G.K.), FAPESC (M.B.P.), and CAPES (K.P.G). R.L.S.F. would like to thank J. Noronha and R.O. Ramos for discussions on related matters.
[1] K. Fukushima, D. E. Kharzeev, and H. J. Warringa, Phys. Rev. D 78, 074033 (2008).

[2] D. E. Kharzeev and H. J. Warringa, Phys. Rev. D 80, 034028 (2009).

[3] D. E. Kharzeev, Nucl. Phys. A 830, 543c (2009).

[4] K. Tuchin, Adv. High Energy Phys. 2013, 490495 (2013).

[5] R. Duncan and C. Thompson, Astrophys. J. 392, L9 (1992).

[6] C. Kouveliotou et al., Nature (London) 393, 235 (1998).

[7] T. Vaschapati, Phys. Lett. B 265, 258 (1991).

[8] Y. Aoki, G. Endrodi, Z. Fodor, S. D. Katz, and K. K. Szabo, Nature (London) 443, 675 (2006).

[9] Y. Aoki, Z. Fodor, S. D. Katz, and K. K. Szabo, Phys. Lett. B 643, 46 (2006).

[10] M. D'Elia, S. Mukherjee, and F. Sanfilippo, Phys. Rev. D 82, 051501 (2010).

[11] E.-M. Ilgenfritz, M. Kalinowski, M. Müller-Preussker, B. Petersson, and A. Schreiber, Phys. Rev. D 85, 114504 (2012).

[12] G. S. Bali, F. Bruckmann, G. Endrödi, Z. Fodor, S. D. Katz, S. Krieg, A. Schäfer, and K. K. Szabó, J. High Energy Phys. 02 (2012) 044.

[13] G. S. Bali, F. Bruckmann, G. Endrödi, Z. Fodor, S. D. Katz, and A. Schäfer, Phys. Rev. D 86, 071502(R) (2012).

[14] S. P. Klevansky and R. H. Lemmer, Phys. Rev. D 39, 3478 (1989).

[15] V. P. Gusynin, V. A. Miransky, and I. A. Shovkovy, Phys. Lett. B 349, 477 (1995).
[16] I. A. Shushpanov and A. V. Smilga, Phys. Lett. B 402, 351 (1997).

[17] E. S. Fraga and A. J. Mizher, Phys. Rev. D 78, 025016 (2008).

[18] A. J. Mizher, M. N. Chernodub, and E. S. Fraga, Phys. Rev. D 82, 105016 (2010).

[19] S. S. Avancini, D. P. Menezes, M. B. Pinto, and C. Providencia, Phys. Rev. D 85, 091901 (2012).

[20] J. O. Andersen and R. Khan, Phys. Rev. D 85, 065026 (2012).

[21] G. N. Ferrari, A. F. Garcia, and M. B. Pinto, Phys. Rev. D 86, 096005 (2012).

[22] E. S. Fraga and L. F. Palhares, Phys. Rev. D 86, 016008 (2012).

[23] D. P. Menezes, M. B. Pinto, S. S. Avancini, A. Pérez Martínez, and C. Providência, Phys. Rev. C 79, 035807 (2009).

[24] V. Skokov, Phys. Rev. D 85, 034026 (2012).

[25] J. O. Andersen and A. Tranberg, J. High Energy Phys. 08 (2012) 002.

[26] K. Fukushima and Y. Hidaka, Phys. Rev. Lett. 110, 031601 (2013).

[27] T. Kojo and N. Su, Phys. Lett. B 720, 192 (2013).

[28] F. Bruckmann, G. Endrodi, and T. G. Kovacs, J. High Energy Phys. 04 (2013) 112.

[29] E. S. Fraga, J. Noronha, and L. F. Palhares, Phys. Rev. D 87, 114014 (2013).

[30] Y. Sakai, T. Sasaki, H. Kouno, and M. Yahiro, Phys. Rev. D 82, 076003 (2010); J. Phys. G 39, 035004 (2012). 
[31] T. Sasaki, Y. Sakai, H. Kouno, and M. Yahiro, Phys. Rev. D 84, 091901(R) (2011).

[32] K. Fukushima, M. Ruggieri, and R. Gatto, Phys. Rev. D 81, 114031 (2010).

[33] M. Ferreira, P. Costa, D. P. Menezes, C. Providência, and N. N. Scoccola, Phys. Rev. D 89, 016002 (2014).

[34] E. S. Fraga, B. W. Mintz, and J. Schaffner-Bielich, Phys. Lett. B 731, 154 (2014).

[35] V. A. Miransky and I. A. Shovkovy, Phys. Rev. D 66, 045006 (2002).

[36] V. Bernard, U.-G. Meissner, and I. Zahed, Phys. Rev. D 36, 819 (1987).

[37] S.-x. Qin, L. Chang, Y.-x. Li, and C. D. Roberts, Phys. Rev. D 84, 014017 (2011).

[38] C. D. Roberts and S. M. Schmidt, Prog. Part. Nucl. Phys. 45, S1 (2000).
[39] Y. Nambu and G. Jona-Lasinio, Phys. Rev. 122, 345 (1961).

[40] M. Buballa, Phys. Rep. 407, 205 (2005).

[41] J.-L. Kneur, M. B. Pinto, and R. O. Ramos, Phys. Rev. C 81, 065205 (2010).

[42] F. Preis, A. Rebhan, and A. Schmitt, J. High Energy Phys. 03 (2011) 033; Lect. Notes Phys. 871, 51 (2013).

[43] E. J. Ferrer, V. de la Incera, I. Portillo, and M. Quiroz, Phys. Rev. D 89, 085034 (2014).

[44] A. Ayala, A. Bashir, A. Raya, and E. Rojas, Phys. Rev. D 73, 105009 (2006).

[45] E. Rojas, A. Ayala, A. Bashir, and A. Raya, Phys. Rev. D 77, 093004 (2008).

[46] P. Watson and H. Reinhardt, Phys. Rev. D 89, 045008 (2014).

[47] N. Mueller, J. A. Bonnet, and C. S. Fischer, Phys. Rev. D 89, 094023 (2014). 